\title{
Hepatic autotaxin overexpression in infants with biliary atresia
}

\author{
Wanvisa Udomsinprasert ${ }^{1,2}$, Paisarn Vejchapipat ${ }^{3}$, Naruemon Klaikeaw ${ }^{4}$, Voranush Chongsrisawat ${ }^{5}$, Yong \\ Poovorawan $^{5}$, Sittisak Honsawek ${ }^{\text {Corresp. } 1}$ \\ ${ }^{1}$ Osteoarthritis and Musculoskeleton Research Unit, Department of Biochemistry, Faculty of Medicine, King Chulalongkorn Memorial Hospital, Thai Red \\ Cross Society, Chulalongkorn University, Bangkok, Thailand \\ 2 Department of Biochemistry, Faculty of Pharmacy, Mahidol University, Bangkok, Thailand \\ 3 Department of Surgery, Faculty of Medicine, King Chulalongkorn Memorial Hospital, Thai Red Cross Society, Chulalongkorn University, Bangkok, Thailand \\ 4 Department of Pathology, Faculty of Medicine, King Chulalongkorn Memorial Hospital, Thai Red Cross Society, Chulalongkorn University, Bangkok, \\ Thailand \\ ${ }^{5}$ Center of Excellence in Clinical Virology, Department of Pediatrics, Faculty of Medicine, King Chulalongkorn Memorial Hospital, Chulalongkorn University, \\ Bangkok, Thailand \\ Corresponding Author: Sittisak Honsawek \\ Email address: sittisak.h@chula.ac.th
}

Background. Autotaxin (ATX) is a secreted glycoprotein that is involved in the development of hepatic fibrogenesis via the enzymatic production of lysophosphatidic acid. The aim of this study was to investigate hepatic expression of ATX in biliary atresia (BA) compared with non-BA liver controls and to examine the association between ATX expression and clinical outcome in BA. Methods. Liver specimens from BA infants $(n=20)$ were compared with samples from infants who underwent liver biopsy for reasons other than BA $(n=14)$ served as controls. Relative mRNA and protein expression of ATX were quantified using real-time polymerase chain reaction (PCR) and immunohistochemistry. Masson's Trichrome staining was performed to determine the degree of liver fibrosis. Results. Quantitative real-time PCR demonstrated overexpression of ATX mRNA in BA livers. In immunohistochemical evaluation, ATX was positively stained on the hepatic parenchyma and the biliary epithelium in BA patients, as compared to non-BA controls. The immunostaining score of ATX in BA livers was also significantly higher than that observed in non-BA livers $(P<0.001)$. Subgroup analysis revealed that ATX expression in the patients with poor outcome was significantly greater than in those with good outcome $(P=0.03)$. Additionally, there was a positive correlation between hepatic ATX expression and Metavir fibrosis stage in BA livers $(r=0.79, P<0.001)$. Discussion. This study found that mRNA and protein expression of ATX was increased in BA livers. High hepatic ATX expressionat the time of Kasai operation was associated with liver fibrosis and outcome in BA, suggesting that ATX may serve a role as a promising biomarker of the prognosis in biliary atresia. 


\section{Manuscript Title:}

\section{Hepatic autotaxin overexpression in infants with biliary atresia}

\section{Authors:}

4 Wanvisa Udomsinprasert ${ }^{1,2}$, Paisarn Vejchapipat $^{3}$, Naruemon Klaikeaw ${ }^{4}$, Voranush

5 Chongsrisawat $^{5}$, Yong Poovorawan ${ }^{5}$, Sittisak Honsawek ${ }^{1, *}$

\section{Affiliations:}

$7{ }^{1}$ Osteoarthritis and Musculoskeleton Research Unit, Department of Biochemistry, Faculty of

8 Medicine, Chulalongkorn University, King Chulalongkorn Memorial Hospital, Thai Red Cross

9 Society, Bangkok, Thailand

10 2Department of Biochemistry, Faculty of Pharmacy, Mahidol University, Bangkok, Thailand

$11{ }^{3}$ Department of Surgery, Faculty of Medicine, Chulalongkorn University, King Chulalongkorn

12 Memorial Hospital, Thai Red Cross Society, Bangkok, Thailand

$13{ }^{4}$ Department of Pathology, Faculty of Medicine, Chulalongkorn University, King Chulalongkorn

14 Memorial Hospital, Thai Red Cross Society, Bangkok, Thailand

$15{ }^{5}$ Center of Excellence in Clinical Virology, Department of Pediatrics, Faculty of Medicine,

16 Chulalongkorn University, King Chulalongkorn Memorial Hospital, Bangkok, Thailand

Corresponding Author:

Sittisak Honsawek, MD, PhD

20 Osteoarthritis and Musculoskeleton Research Unit,

Department of Biochemistry,

22 Faculty of Medicine, Chulalongkorn University, 
23 King Chulalongkorn Memorial Hospital, Thai Red Cross Society, Bangkok, Thailand

24 E-mail: sittisak.h@chula.ac.th

\section{Abstract}

Background. Autotaxin (ATX) is a secreted glycoprotein that is involved in the development of hepatic fibrogenesis via the enzymatic production of lysophosphatidic acid. The aim of this study was to investigate hepatic expression of ATX in biliary atresia (BA) compared with non-BA liver controls and to examine the association between ATX expression and clinical outcome in BA.

Methods. Liver specimens from BA infants $(n=20)$ were compared with samples from infants who underwent liver biopsy for reasons other than BA $(n=14)$ served as controls. Relative mRNA and protein expression of ATX were quantified using real-time polymerase chain reaction (PCR) and immunohistochemistry. Masson's Trichrome staining was performed to determine the degree of liver fibrosis.

Results. Quantitative real-time PCR demonstrated overexpression of $A T X$ mRNA in BA livers. In immunohistochemical evaluation, ATX was positively stained on the hepatic parenchyma and the biliary epithelium in BA patients, as compared to non-BA controls. The immunostaining score of ATX in BA livers was also significantly higher than that observed in non-BA livers $(P<0.001)$. Subgroup analysis revealed that ATX expression in the patients with poor outcome was significantly greater than in those with good outcome $(P=0.03)$. Additionally, there was a

42 positive correlation between hepatic ATX expression and Metavir fibrosis stage in BA livers $43 \quad(r=0.79, P<0.001)$.

44 Discussion. This study found that mRNA and protein expression of ATX was increased in BA 45 livers. High hepatic ATX expression at the time of Kasai operation was associated with liver 
46 fibrosis and outcome in BA, suggesting that ATX may serve a role as a promising biomarker of

47 the prognosis in biliary atresia.

48 Keywords: Biliary atresia; autotaxin; immunohistochemistry; liver; quantitative real-time

49 polymerase chain reaction

50

51

52

\section{Introduction}

Biliary atresia (BA) is a neonatal cholestasis disease that is characterized by fibrosclerosing and inflammatory obliteration of the biliary tracts, which leads to progressive liver damage (Hartley et al., 2009). Kasai portoenterostomy (KPE), the primary treatment for BA, establishes good bile flow and facilitates long-term survival. However and even after timely KPE, a number of infants are at risk of developing new biliary obstruction that could lead to chronic cholestasis, increased fibrosis, cirrhosis, and eventually to end-stage liver disease. As such, BA is the leading cause of liver transplantation in children. Although the precise pathogenesis of BA remains elusive, possible etiologies include viral infection, toxins, chronic inflammatory or immune-mediated bile duct injury, and abnormalities in bile duct development (Bezerra, 2005). Increased understanding of what causes inflammatory cholangiopathy in BA could lead to therapies aimed at protecting the intrahepatic biliary system from inflammation-mediated fibrosis. However, the molecular mechanisms involved in the pathogenesis of liver fibrosis in BA have not yet been fully and clearly established. Hepatic fibrosis is a reversible physiologic and pathologic event, and the possible role of cytokine-mediated pathogenesis in this disorder is of great interest to many researchers.

Autotaxin [ATX; ectonucleotide pyrophosphatase/phosphodiesterase family member 2 (ENPP2)] is a secreted lysophospholipase D that generates the lipid mediator lysophosphatidic acid (LPA) from extracellular lysophospholipids - predominantly from lysophosphatidylcholine 
69 (Tokumura et al., 2002). ATX-LPA signaling has been implicated in multiple biological and

70 pathophysiological processes, including vasculogenesis, cholestatic pruritus, tumor progression,

71 and fibrosis via 6 distinct G-protein-coupled LPA receptors (LPAR1-6) (Umezu-Goto et al.,

72 2002). Ikeda et al., identified a potential link between the ATX-LPA axis and liver fibrosis when

73 they found that intradermal LPA induces hepatic stellate cell (HSC) proliferation, stimulates

74 their contraction, and inhibits their apoptosis. HSCs are known as prototypic profibrogenic cells

75 in the hepatic parenchyma (Ikeda et al., 2003). After transformation into myofibroblasts in

76 response to a liver injury, HSCs start to produce abundant extracellular matrices and

77 profibrogenic cytokines, such as ATX-derived LPA. In addition, both LPA and ATX

concentrations were increased in chronic hepatitis $\mathrm{C}$ patients with liver fibrosis (Watanabe et al.,

79 2007). The $A T X-L P A$ axis has also been reported to be up-regulated in human hepatocellular carcinoma (Park et al., 2011; Wu et al., 2010), thereby establishing the possible influence of ATX in inflammation-related hepatic fibrosis disorders like biliary atresia.

Although circulating ATX levels have been shown to be associated with liver fibrosis, there is limited information on ATX expression in liver tissue and regarding the association between ATX expression and BA outcomes. Accordingly, the aim of this study were to investigate mRNA and protein expression of ATX in liver tissues from BA patients compared with non-BA controls and to evaluate whether hepatic ATX expression is associated with 87 outcome parameters in BA infants.

Materials and Methods 
91 The study protocol conformed to the ethical standards outlined in the Declaration of Helsinki and

92 was approved by the Institutional Review Board of the Faculty of Medicine, Chulalongkorn

93 University (IRB No. 549/57). All parents of children were fully informed regarding the study

94 protocol and procedures prior to the children entering the study. Written informed consent was

95 obtained from the participants' parents.

96 Perioperative liver biopsies were obtained from 20 BA infants at the time of KPE and 14

97 non-BA patients at the Department of Surgery, King Chulalongkorn Memorial Hospital during

98 the July 2005 to July 2007 study period. All BA infants were invited to participate in this study

99 based on the following criteria: (1) diagnosis of type 3 (uncorrectable) isolated BA and they

100 underwent Kasai procedure; (2) availability of clinical details and long term follow-up after

101 surgery; and (3) availability of archived glass slides or paraffin blocks of wedge liver specimens

102 taken at KPE. Infants diagnosed with BA or non-BA were included based on clinical,

103 cholangiographic, and histologic findings. Non-BA patients with no history of immune-mediated

104 diseases served as controls. Non-BA control samples were collected from 6 patients with

105 choledochal cyst, 4 patients with thalassemia, 3 patients with neuroblastoma, and 1 patient with

106 hepatoblastoma. Liver biopsies taken from non-BA controls were obtained during procedures

107 that were required for medical reasons. Liver specimens from age-matched healthy controls

108 could not be obtained due to ethical concerns about harvesting liver tissue from healthy infants.

109 Demographic and clinical data collected at the time of KPE included age, albumin, total

110 bilirubin (TB), and alanine aminotransferase (ALT). Laboratory investigations were performed

111 on a Roche Hitachi 912 chemistry analyzer (Roche Diagnostics, Basel, Switzerland). In order to

112 associate ATX hepatic expression with outcome at 6 months post-Kasai in BA, the infants were

113 divided into good outcome and poor outcome based on their levels of serum TB, ALT, and 
114 clinical findings. Nine patients with good outcome had good bile flow after KPE. The stool color

115 turned from pale to yellowish for 6 months following successful surgery. The serum TB returned

116 to normal with satisfactory liver function (TB $<2 \mathrm{mg} / \mathrm{dL}, \mathrm{ALT}<100 \mathrm{IU} / \mathrm{L})$. Another 11 patients

117 with poor outcome had cholestasis after 6 months KPE and severe liver dysfunction (TB $\geq 2$

$118 \mathrm{mg} / \mathrm{dL}, \mathrm{ALT} \geq 100 \mathrm{IU} / \mathrm{L})$.

$119 R N A$ extraction and quantitative real time-PCR for $m R N A$ expression of $A T X$

120 Of 20 BA infants and 14 non-BA controls, 15 BA livers and 5 non-BA liver specimens

121 (choledochal cyst) were snap-frozen in liquid nitrogen-cooled isopentane and stored at $-80^{\circ} \mathrm{C}$

122 and only available for $A T X$ mRNA expression. Total RNA was isolated from liver biopsies using

123 RNeasy Mini Kit (Qiagen, Hilden, Germany) with cDNA that was reverse transcribed using

124 TaqMan Reverse Transcription Reagents (Applied Biosystems, Inc., Foster City, CA, USA).

125 Real-time PCR was performed using QPCR Green Master Mix HRox (biotechrabbit GmbH,

126 Hennigsdorf, Germany) on StepOnePlus Real-Time PCR System (Applied Biosystems, Inc.,

127 Foster City, CA, USA). Primers used for $A T X$ and glyceraldehyde 3-phosphate dehydrogenase

$128(G A D P H)$ amplification were, as follows: $A T X$ forward primer 5'-

129 CGTGGCTGGGAGTGTACTAA-3'; ATX reverse primer 5'-AGAGTGTGTGCCACAAGACC-

130 3', as previously described (Kondo et al., 2014); GADPH forward primer 5'-

131 GTGAAGGTCGGAGTCAACGG-3'; and, GADPH reverse primer 5'-

132 TCAATGAAGGGGTCATTGATGG-3'. Real-time PCR was performed, as follows: (initial 133 step) $95^{\circ} \mathrm{C}$ for $10 \mathrm{~min}$, followed by 40 cycles of $95^{\circ} \mathrm{C}$ for $15 \mathrm{sec}$, and then $60^{\circ} \mathrm{C}$ for $1 \mathrm{~min}$.

134 Relative mRNA expression of $A T X$ was normalized to $G A D P H$ as an internal control and was

135 determined using $2^{-\Delta \Delta \mathrm{Ct}}$ method.

136 Masson's Trichrome staining 
137 Masson's Trichrome staining was conducted according to the manufacturer's protocol (Genmed

138 Scientifics, Wilmington, DE). The collagen fiber was stained blue, the nuclei were stained black,

139 and the background was stained red. Liver fibrosis was evaluated according to the Metavir

140 grading system (Bedossa et al., 1996) as follows: F0, no fibrosis; F1, mild fibrosis in the portal

141 area; F2, mild bridging fibrosis in the adjacent portal area; F3, severe bridging fibrosis in the

142 adjacent portal area; and F4, cirrhosis and annular fibrosis with nodule formation.

143 Immunohistochemical analysis for protein expression of ATX

144 All liver specimens of 20 BA patients and 14 non-BA controls were paraffin-embedded and then

145 sectioned according to standard protocols. Routine staining with hematoxylin and eosin, and

146 immunohistochemical staining with antibodies was performed to detect protein expression of

147 ATX (Merck Millipore, Darmstadt, Germany). For ATX staining, cells with brown stained

148 cytoplasm were scored as positive. All tissue sections were analyzed by a pathologist who was

149 blinded to patient clinical status and diagnosis. Immunoreactivity of ATX in the biliary

150 epithelium and the parenchyma was semi-quantitatively analyzed for percentage of positive cells

151 and intensity of staining. A percentage of positive cells $<1 \%$ was scored as $0 ; 1 \%-25 \%$ as 1 ;

$152>25 \%-50 \%$ as $2 ;>50 \%-75 \%$ as 3 ; and, $>75 \%$ as 4 . Intensity of ATX immunostaining was

153 determined using the following staining scores: $0=$ no staining; $1=$ weak staining; $2=$ moderate

154 staining; and 3 = strong staining. Final results were scored by the total score [total scores $=$

$155(($ score of positive cell + score of intensity $) \times 100) /$ maximum score of both parameters]. Using the

156 aforementioned staining scores, the positive areas of ATX positive cells were determined by

157 measuring five randomly selected microscopic fields $(400 \times)$ on each slide.

158 Statistical analysis 
159 All statistical analyses were performed using SPSS Statistics version 22.0 (SPSS, Inc., Chicago, 160 IL, USA). Demographic and clinical characteristics between groups were evaluated using Chi161 square tests and unpaired Student's $t$-tests where appropriate. The comparisons of ATX 162 expression between groups were performed by Mann-Whitney $U$-tests. Correlations were analyzed by Spearman's rank correlation. The curves for survival were drawn according to the Kaplan-Meier analysis with end points of death. The differences of survival curves were $P$-value $<0.05$ was considered to be statistically significant for differences and correlations.

\section{Results}

\section{Clinical characteristics of study participants}

Baseline characteristics of BA infants and non-BA controls are summarized in Table 1 . There was no statistically significant difference in gender ratio between BA patients and non-BA controls. All non-BA participants had no clinical jaundice. The diagnosis of non-BA subjects included 6 choledochal cysts, 4 thalassemias, 3 neuroblastomas, and 1 hepatoblastoma.

\section{Relative mRNA expression of $A T X$}

177 To identify mRNA expression of $A T X$ in infants with BA, relative $A T X$ mRNA expression was 178 quantified by real-time polymerase chain reaction (PCR) in liver biopsies from BA patients $179(n=15)$ and non-BA controls $(n=5)$. Relative $A T X$ mRNA expression was found to be 180 significantly higher in BA livers than non-BA liver controls $(P<0.05)$ (Figures 1A; B). 
182 Immunohistochemical evaluation for ATX protein expression was performed in both BA and

183 non-BA liver tissues. Representative immunohistochemical findings of ATX are illustrated in

184 Figure 2. In congenital BA liver specimens, overexpression of ATX was detectable in the hepatic

185 parenchyma, biliary epithelial cells, and cells of the surrounding connective tissue. In contrast,

186 ATX expression was only scarcely evident in non-BA control livers, being demonstrated as faint

187 cytoplasmic staining (Figure 2A). The distribution of ATX in positive cells was classified as

188 cytoplasm-localized pattern.

In order to compare expression levels of ATX protein between BA patients and non-BA

190

191

192

193

194

195

196

197

198

199

200

201

202

203

controls, staining intensity and percentage of ATX positive cells were assessed by visual scoring

method. In BA livers at the time of KPE, hepatic ATX protein expression was significantly

higher than that in non-BA controls when measured by the total score of staining on histologic

liver sections $(P<0.001)$ (Figure 3A). There was no association between ATX mRNA and

protein expression in BA livers.

Hepatic ATX protein expression in BA subgroups

To determine whether hepatic ATX protein expression would be associated with poor outcomes in BA patients, we classified BA children according serum TB, liver enzymes, and clinical findings at 6 months post-Kasai into patients with poor outcome $(n=11)$ and patients with good outcome $(n=9)$. Table 2 demonstrates the clinical characteristics of the BA subgroups based on clinical outcome at 6 months post-operation. Subsequent analysis demonstrated the mean immunoreactive score of ATX protein expression in BA patients with poor outcome was significantly greater than in patients with good outcome $(P=0.03)$ (Figure 3B). We further analyzed the correlation between hepatic ATX protein expression and markers of liver function 
204 in BA patients. The results showed no association between hepatic ATX protein expression and

205 liver function parameters in BA infants at the time of KPE.

206 Increased hepatic ATX protein expression and liver fibrosis

207 The portal areas illustrated various degrees of fibrosis, but fibrous septa were generally broad

208 with lobular extension (Figure 4A). The liver fibrosis grade was F0 in 5 cases, F1 in 6 cases, F2

209 in 7 cases, and F3 in 2 cases. As shown in Figure 4B, hepatic ATX protein expression was

210 positively correlated with Metavir fibrosis stage in BA $(r=0.79, P<0.001)$.

\section{Survival curve analysis}

212 We performed Kaplan-Meier analysis to investigate the overall survival curve of all 20 BA

213

214

215

216

217

218

219

220

221

222

223

224

225

226

children. The 10-year survival rate with native livers of all BA children were estimated $80 \%$, as

shown in Figure 5A. When stratified into low and high ATX protein expression using the cut-off value of $50 \%$, the overall survival rates at 10 years were $91.7 \%$ for those with low expression and $62.5 \%$ for those with high expression. Survival rate was greater in BA patients with low ATX expression than those with high ATX expression (log-rank, $X^{2}=2.17, P=0.14$ ) (Figure 5B).

\section{Discussion}

Despite extensive research efforts, the understanding of mechanisms that regulate biliary atresia (BA) progression following Kasai portoenterostomy (KPE) remains unclear. Biliary atresia causes rapidly progressive liver fibrosis and cirrhosis in neonates, and is the most common indication for liver transplantation in children. Although the precise cause of liver fibrosis in BA remains unclear, several cytokines have been implicated in the regulation of hepatic fibrogenesis (Kanzler et al., 1999; Williams et al., 2000; Farrington et al., 2010; Xiao et al., 2015;

Iordanskaia et al., 2015; Klemann et al., 2016). In a previous study, we reported association 
227 between elevation of circulating autotaxin (ATX) and poor outcomes in BA patients - especially

228 severity of fibrosis (Udomsinprasert et al., 2015). This is important evidence that supports the

229 hypothesis that ATX may serve a role as a potential biomarker of the prognosis in BA. In the

230 present study, we investigated mRNA and protein expression of ATX in liver biopsies from BA

231 infants compared with non-BA controls, and we found up-regulated ATX mRNA in BA patients.

232 We then performed immunohistochemical analysis to determine protein expression of ATX and

233 found an intense increase in ATX staining in BA infants, predominantly in the hepatic

234 parenchyma and biliary epithelium at the time of KPE.

235 The biological outcome of ATX has been shown to induce a variety of inflammatory

236

237

238

239

240

241

242

243

244

245

246

247

248

249

phenomena via LPA activity, and its role in disease pathophysiology has been verified in several diseases (Umezu-Goto et al., 2002), making ATX-derived LPA signaling an attractive therapy. Indeed, emerging evidence suggests that the liver is the main source of ATX metabolism in both human and animal models (Ikeda \& Yatomi, 2012). Additionally, ATX expression has been detected on all types of liver cells, including the biliary epithelium (Kremer et al., 2010). This suggests the possibility of ATX having a regulatory role in the liver. Our result demonstrated that $A T X$ mRNA expression was up-regulated in the livers of BA infants when compared with nonBA liver controls. This finding is consistent with a previous investigation that reported overexpression of $A T X$ in liver tissues of patients with hepatocellular carcinoma (HCC) (Cooper et al., 2007), suggesting that up-regulated $A T X$ expression is associated with hepatic damage and liver fibrosis. In addition to up-regulation of $A T X$ mRNA expression in the livers of BA infants, an increase in hepatic protein expression of ATX was also demonstrated. A recently published report also confirmed that overexpression of ATX protein was specifically associated with inflammation and cirrhosis in HCC patients ( Wu et al., 2010). This was in agreement with our 
250 finding, implying that ATX may play a role in inflammation that is related to progressive BA.

251 Furthermore, our immunohistochemistry data demonstrated positive cytoplasmic ATX

252 expression in inflammatory cells and biliary epithelial cells. Indeed, hepatic ATX expression

253 varied between different stages of cholestasis. This study also revealed that there was a positive

254 correlation between hepatic ATX expression and the degree of fibrosis, suggesting the possibility

255 of ATX as a predictive tool for discriminating between good and poor prognosis of clinical

256 outcome in postoperative BA. Consistent with our finding, Wunsch, et al. have demonstrated

257 elevated ATX expression in chronic cholestatic diseases (Wunsch et al., 2016). In addition, a

258 recent study revealed that high ATX expression was detected in hepatocellular carcinoma and

259 was correlated with histological grade and survival rate (Memet et al., 2017). Rather, ATX might

260 be associated with the nature of BA disease itself. Thus, it is reasonable to postulate that

261 increased expression of ATX in BA livers might reflect a defensive response by the body to fight

262 against hepatic impairment, or may simply be a compensatory response to ATX, which leads to

263 its compensatory up-regulation.

264 The potential significance of elevated ATX expression in BA remains unclear. The

265 aberrant production of ATX may result in the altered activation of LPA signaling pathways via

266 G-protein-coupled LPA-receptors, and may not be limited to activation of signaling-associated

267 cell proliferation, migration, and apoptosis. Hepatic stellate cells (HSCs) are known to play a

268 major role in the fibrotic process in the liver and they may contribute to the prognosis of BA. For

269 this reason, multiple factors with potentially fibrogenic activities in the liver have been evaluated

270 due to their effects on HSC activation and apoptosis. Regarding the potential effect of ATX-

271 mediated LPA on HSCs, LPA has been shown to stimulate the contractility of HSCs and to

272 inhibit their apoptosis via Rho/Rho kinase activation (Ikeda et al., 2003; Yanase et al., 2003). 
273 Although ATX may not play a primary role in the pathogenesis of liver fibrosis, it may

274 accelerate fibrogenesis by stimulating the proliferation of HSCs in patients with liver fibrosis via

275 its ability to produce LPA. This hypothesis has been supported by the recent observation that

276 specific ATX transgenic overexpression and/or gene disruption from hepatocytes in mice models

277 of chronic liver injury established a liver profibrotic role for ATX/LPA (Kaffe et al., 2017). A

278 more recent study by Bain, et al. found that a selective ATX inhibitor (PAT-505) markedly

279 reduced liver fibrosis in mouse models (Bain et al., 2017). From those findings, we observe that

280 hepatic ATX expression was associated with an adverse clinical outcome in BA, which lends

281 support to the hypothesis that inhibiting ATX as part of an antifibrotic model could serve as a novel therapeutic approach for treatment of hepatic fibrosis in BA patients. Taken together, the aforementioned findings suggest that the aberrant expression of ATX may be used as a promising biomarker for predicting the progression and prognosis of biliary atresia after Kasai portoenterostomy. Further experiments that isolate biliary epithelial cells and HSCs from BA livers will be required to determine the precise biological and pathological significance of the findings and observations presented in this report.

This study has some mentionable limitations. The most notable limitation is the fact that we were unable to obtain age-matched liver tissue from healthy infants due to ethical considerations. The limited availability of frozen liver biopsies from non-BA controls could have posed significant challenges to the study. Second, the sample size of our study population is relatively small. This is due, in large part, to the fact that BA is a relatively rare disorder. The limited number of subjects makes it challenging to show significant correlations of all parameters in BA patients. Future larger scale, multicenter studies should be conducted to verify our conclusions. Another caveat is the lack of data regarding the circulating ATX levels, total 
296

297

298

299

300

301

302

303

304

305

306

307

308

309

310

311

312

313

314

315

316

317

318

serum bile salt levels, and cholestatic pruritis. We recognize that these could be addressed by

prospective longitudinal multicenter cohorts. Further research of costaining on BA liver specimens will identify the cellular fractions expressing ATX. Finally, the causal association between hepatic ATX expression and BA was not fully addressed in the study. Additional research is required to evaluate whether increased hepatic ATX expression is causally related to progressive BA or whether it is simply a compensatory response to the disease.

\section{Conclusions}

The current study presents evidence of the up-regulation of $A T X$ mRNA expression in liver specimens of BA patients, as compared to specimens from livers of non-BA controls. ATX was expressed not only in the hepatic parenchyma, but also in biliary epithelial cells of BA infants at the time of KPE. These findings suggest that ATX expression could be related to liver fibrosis and outcome in biliary atresia. Further investigations examining the possible effect of selective ATX inhibitors on inflammation and progression of liver fibrosis in BA are needed for the development of non-transplant therapeutic strategies to prevent the progression of this devastating disease in affected infants.

\section{Acknowledgements}

The authors gratefully acknowledge Center of Excellence in Clinical Virology and Chulalongkorn Medical Research Center (ChulaMRC) for providing research facilities, and Preecha Ruangvejvorachai, Thamonwan Woraruthai, and Dong Zhan for technical assistance.

We thank Kevin P. Jones for reviewing and proof-reading the manuscript.

\section{References}

Bain G, Shannon KE, Huang F, Darlington J, Goulet L, Prodanovich P, Ma GL, Santini AM, Stein AJ, Lonergan D, King CD, Calderon I, Lai A, Hutchinson JH, Evans JF. 2017. 
Selective Inhibition of Autotaxin Is Efficacious in Mouse Models of Liver Fibrosis. Journal of Pharmacology and Experimental Therapeutics 360:1-13 DOI 10.1124/jpet.116.237156.

Bedossa P, Poynard T. 1996. An algorithm for the grading of activity in chronic hepatitis C. The METAVIR Cooperative Study Group. Hepatology 24:289-293 DOI 10.1002/hep.510240201.

Bezerra JA. 2005. Potential etiologies of biliary atresia. Pediatric Transplantation 9:646-651 DOI: $10.1111 / \mathrm{j} .1399-3046.2005 .00350$.

Cooper AB, Wu J, Lu D, Maluccio MA. 2007. Is autotaxin (ENPP2) the link between hepatitis C and hepatocellular cancer? Journal of Gastrointestinal Surgery 1:1628-1634 DOI 10.1053/j.gastro.2010.05.009.

Farrington C, Novak D, Liu C, Haafiz AB. 2010. Immunohistochemical localization of transforming growth factor $\beta-1$ and its relationship with collagen expression in advanced liver fibrosis due to biliary atresia. Clinical and Experimental Gastroenterology 3:185-191 DOI 10.2147/CEG.S14220.

Hartley JL, Davenport M, Kelly DA. 2009. Biliary atresia. Lancet 374:1704-1713 DOI: 10.1016/S0140-6736(09)60946-6.

Ikeda H, Nagashima K, Yanase M, Tomiya T, Arai M, Inoue Y, Tejima K, Nishikawa T, Omata M, Kimura S, Fujiwara K. 2003. Involvement of Rho/Rho kinase pathway in regulation of apoptosis in rat hepatic stellate cells. American Journal of Physiology-Gastrointestinal and Liver Physiology 285:G880-886 DOI 10.1152/ajpgi.00039.2003.

Ikeda H, Yatomi Y. 2012. Autotaxin in liver fibrosis. Clinica Chimica Acta 413:1817-1821 DOI 10.1016/j.cca.2012.07.014. 
342 Iordanskaia T, Malesevic M, Fischer G, Pushkarsky T, Bukrinsky M, Nadler E. 2015. Targeting

343 extracellular cyclophilins ameliorates disease progression in experimental biliary atresia.

$344 \quad$ Molecular Medicine 21:657-664.

345 Kaffe E, Katsifa A, Xylourgidis N, Ninou I, Zannikou M, Harokopos V, Foka P, Dimitriadis A,

346 Evangelou K, Moulas AN, Georgopoulou U, Gorgoulis VG, Dalekos GN, Aidinis V. 2017.

347 Hepatocyte Autotaxin expression promotes liver fibrosis and cancer. Hepatology 65:1369-

$348 \quad 1383$ DOI $10.1002 /$ hep.28973.

349 Kanzler S, Lohse AW, Keil A, Henninger J, Dienes HP, Schirmacher P, Rose-John S, zum

350 Büschenfelde KH, Blessing M. 1999. TGF-beta1 in liver fibrosis: an inducible transgenic

351 mouse model to study liver fibrogenesis. American Journal of Physiology 276:G1059-

$352 \quad 1068$.

353 Klemann C, Schröder A, Dreier A, Möhn N, Dippel S, Winterberg T, Wilde A, Yu Y, Thorenz

354 A, Gueler F, Jörns A, Tolosa E, Leonhardt J, Haas JD, Prinz I, Vieten G, Petersen C,

$355 \quad$ Kuebler JF. 2016. Interleukin 17, Produced by $\gamma \delta$ T Cells, Contributes to Hepatic

356 Inflammation in a Mouse Model of Biliary Atresia and Is Increased in Livers of Patients.

357 Gastroenterology 150:229-241 DOI 10.1053/j.gastro.2015.09.008.

358 Kondo M, Ishizawa T, Enooku K, Tokuhara Y, Ohkawa R, Uranbileg B, Nakagawa H, Tateishi

359 R, Yoshida H, Kokudo N, Koike K, Yatomi Y, Ikeda H. 2014. Increased serum autotaxin

360 levels in hepatocellular carcinoma patients were caused by background liver fibrosis but

361 not by carcinoma. Clinica Chimica Acta 433:128-134 DOI 10.1016/j.cca.2014.03.006.

362 Kremer AE, Martens JJ, Kulik W, Ruëff F, Kuiper EM, van Buuren HR, van Erpecum KJ,

363 Kondrackiene J, Prieto J, Rust C, Geenes VL, Williamson C, Moolenaar WH, Beuers U, 
364

365

366

367

368

369

370

371

372

373

374

375

Oude Elferink RP. 2010. Lysophosphatidic acidis a potential mediator of cholestatic pruritus. Gastroenterology 139:1008-1018 DOI 10.1053/j.gastro.2010.05.009.

Memet I, Tsalkidou E, Tsaroucha AK, Lambropoulou M, Chatzaki E, Trypsianis G, Schizas D, Pitiakoudis M, Simopoulos C. 2017. Autotaxin expression in hepatocellular carcinoma. Journal of Investigative Surgery 9:1-7 DOI 10.1080/08941939.2017.1331280.

Park SY, Jeong KJ, Panupinthu N, Yu S, Lee J, Han JW, Kim JM, Lee JS, Kang J, Park CG, Mills GB, Lee HY. 2011. Lysophosphatidic acid augments human hepatocellular carcinoma cell invasion through LPA1 receptor and MMP-9 expression. Oncogene 30:1351-1359 DOI 10.1038/onc.2010.517.

Tokumura A, Majima E, Kariya Y, Tominaga K, Kogure K, Yasuda K, Fukuzawa K. 2002. Identification of human plasma lysophospholipase D, a lysophosphatidic acid-producing enzyme, as autotaxin, a multifunctional phosphodiesterase. Journal of Biological Chemistry 18:39436-39442 DOI 10.1074/jbc.M205623200.

Udomsinprasert W, Honsawek S, Anomasiri W, Chongsrisawat V, Vejchapipat P, Poovorawan Y. 2015. Serum autotaxin levels correlate with hepatic dysfunction and severity in postoperative biliary atresia. Biomarkers 20:89-94 DOI 10.3109/1354750X.2014.994564.

Umezu-Goto M, Kishi Y, Taira A, Hama K, Dohmae N, Takio K, Yamori T, Mills GB, Inoue K, Aoki J, Arai H. 2002. Autotaxin has lysophospholipase D activity leading to tumor cell growth and motility by lysophosphatidic acid production. Journal of Cell Biology 22:227233 DOI 10.1083/jcb.200204026.

Watanabe N, Ikeda H, Nakamura K, Ohkawa R, Kume Y, Aoki J, Hama K, Okudaira S, Tanaka M, Tomiya T, Yanase M, Tejima K, Nishikawa T, Arai M, Arai H, Omata M, Fujiwara K, Yatomi Y. 2007. Both plasma lysophosphatidic acid and serum autotaxin levels are 
387

388

389

390

391

392

393

402

403

404

405

406

407

408

increased in chronic hepatitis C. Journal of Clinical Gastroenterology 41:616-623 DOI 10.1097/01.mcg.0000225642.90898.0e.

Williams EJ, Gaça MD, Brigstock DR, Arthur MJ, Benyon RC. 2000. Increased expression of connective tissue growth factor in fibrotic human liver and in activated hepatic stellate cells. Journal of Hepatology 32:754-761.

Wu JM, Xu Y, Skill NJ, Sheng H, Zhao Z, Yu M, Saxena R, Maluccio MA. 2010. Autotaxin expression and its connection with the TNF-alpha-NF-kappaB axis in human hepatocellular carcinoma. Molecular Cancer 9:71 DOI 10.1186/1476-4598-9-71.

Wunsch E, Krawczyk M, Milkiewicz M, Trottier J, Barbier O, Neurath MF, Lammert F, Kremer AE, Milkiewicz P. 2016. Serum Autotaxin is a Marker of the Severity of Liver Injury and Overall Survival in Patients with Cholestatic Liver Diseases. Scientific Reports 6:30847 DOI 10.1038/srep30847.

Xiao Y, Zhou Y, Chen Y, Zhou K, Wen J, Wang Y, Wang J, Cai W. 2015. The expression of epithelial-mesenchymal transition-related proteins in biliary epithelial cells is associated with liver fibrosis in biliary atresia. Pediatric Research 77:310-315 DOI 10.1038/pr.2014.181.

Yanase M, Ikeda H, Ogata I, Matsui A, Noiri E, Tomiya T, Arai M, Inoue Y, Tejima K, Nagashima K, Nishikawa T, Shibata M, Ikebe M, Rojkind M, Fujiwara K. 2003. Functional diversity between Rho-kinase- and MLCK-mediated cytoskeletal actions in a myofibroblast-like hepatic stellate cell line. Biochemical and Biophysical Research Communications 305:223-228. 
Figure 1

Relative ATX mRNA expression between BA livers and non-BA liver controls.

(A) Up-regulated mRNA expression of ATX normalized by GAPDH in livers from BA infants. (B)

Representative gel of ATX and GAPDH products from real-time PCR analysis. Abbreviations:

M, molecular weight marker; NC, non-template control.

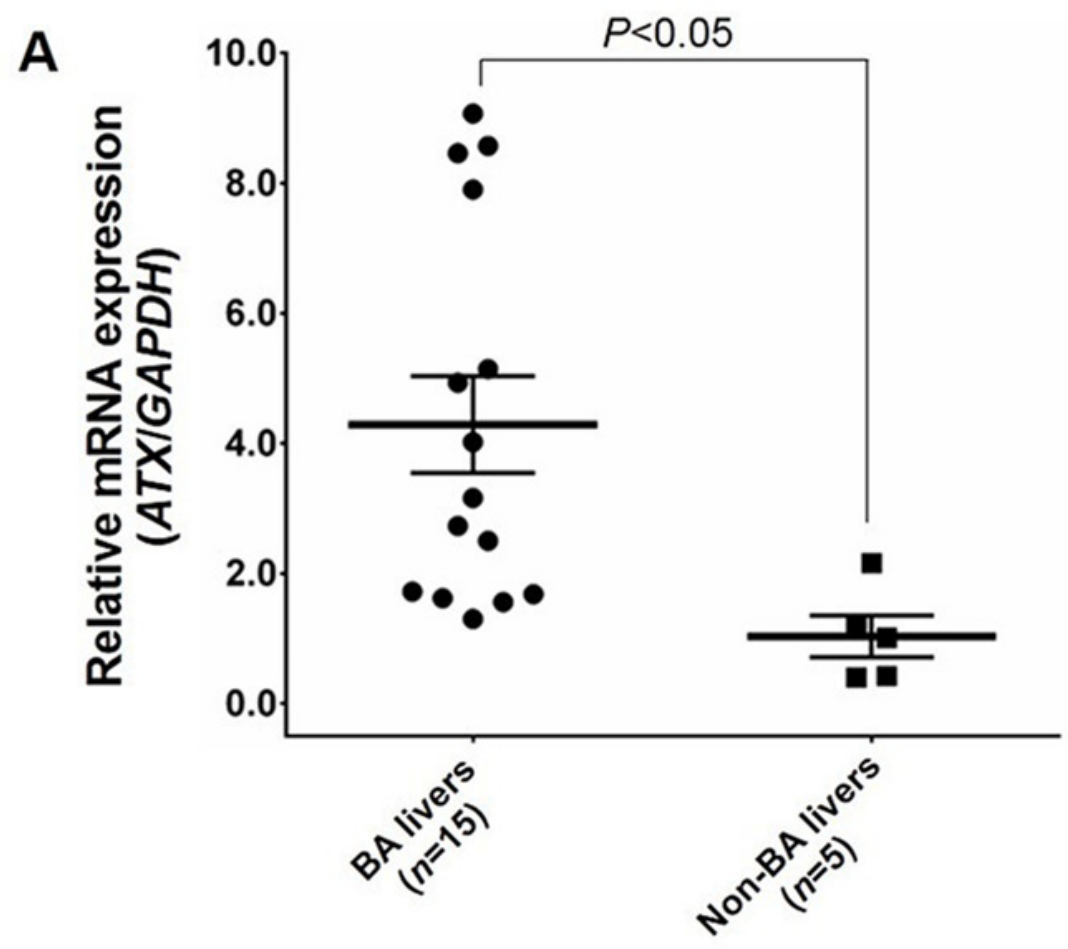

B

BA livers

Non-BA livers

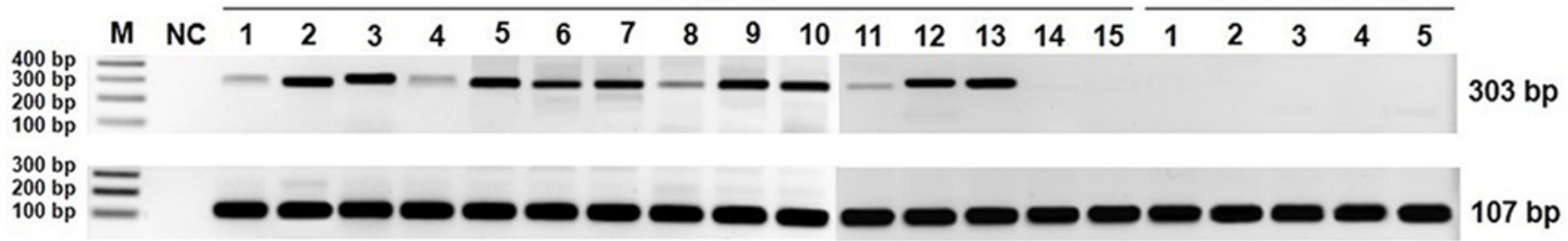




\section{Figure 2}

Immunohistochemical staining for ATX protein expression.

Specific staining of ATX protein is represented by brown coloration. Expression of ATX in BA livers was observed mostly in the hepatic parenchyma (arrows) and biliary epithelium (arrowheads). ATX staining scores were defined, as follows. (A) $0=$ no expression of ATX in a liver used as a control. (B) $1=$ mild expression of ATX in BA liver. (C) $2=$ moderate expression of ATX in BA liver. (D) 3 = strong expression of ATX in BA liver. (Original magnifications $400 x)$.
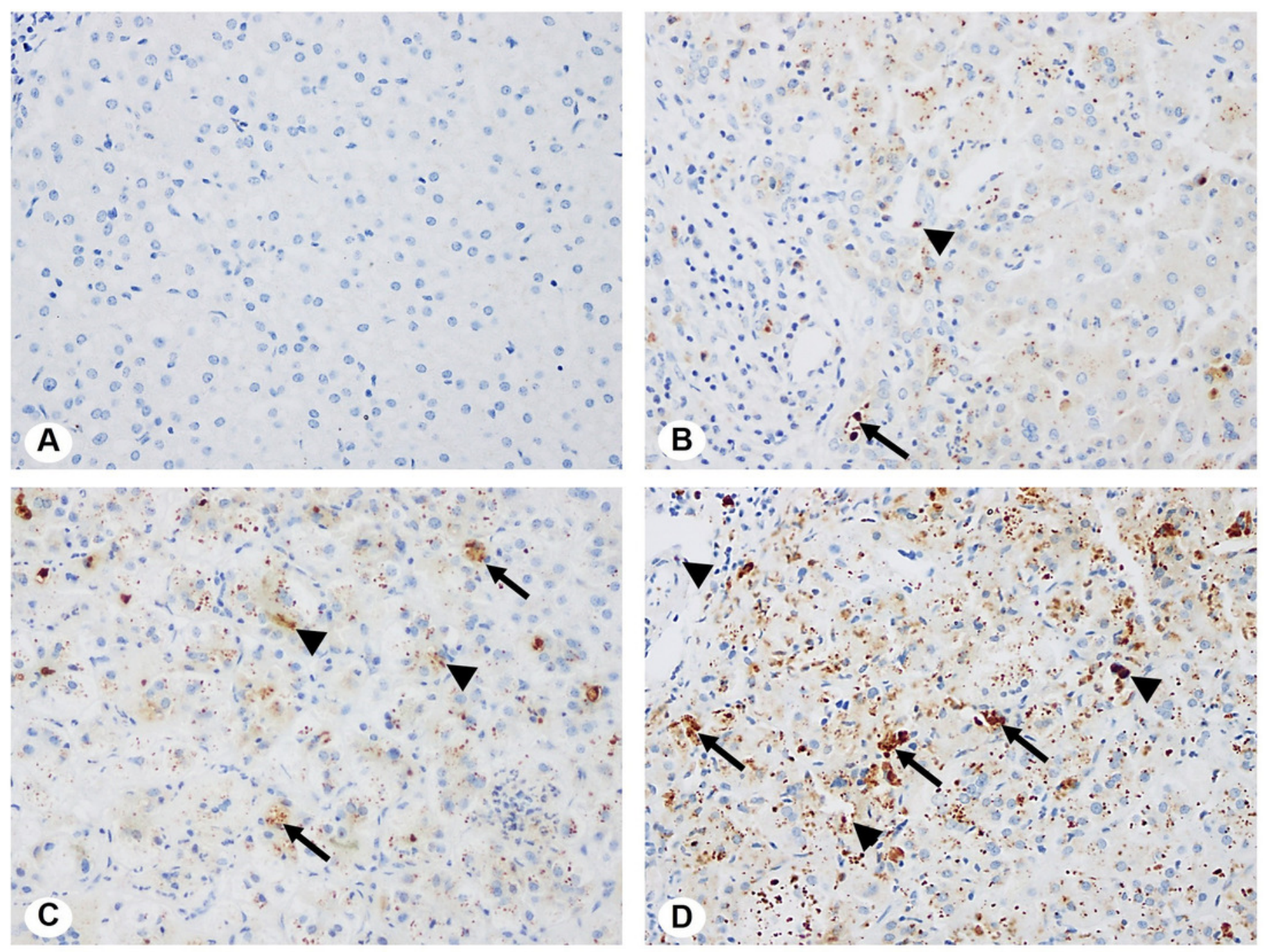
Figure 3

Hepatic ATX protein expression in study subjects between different groups.

(A) Hepatic ATX expression in BA patients and non-BA patients. (B) Hepatic ATX expression in BA patients with poor outcome and good outcome.
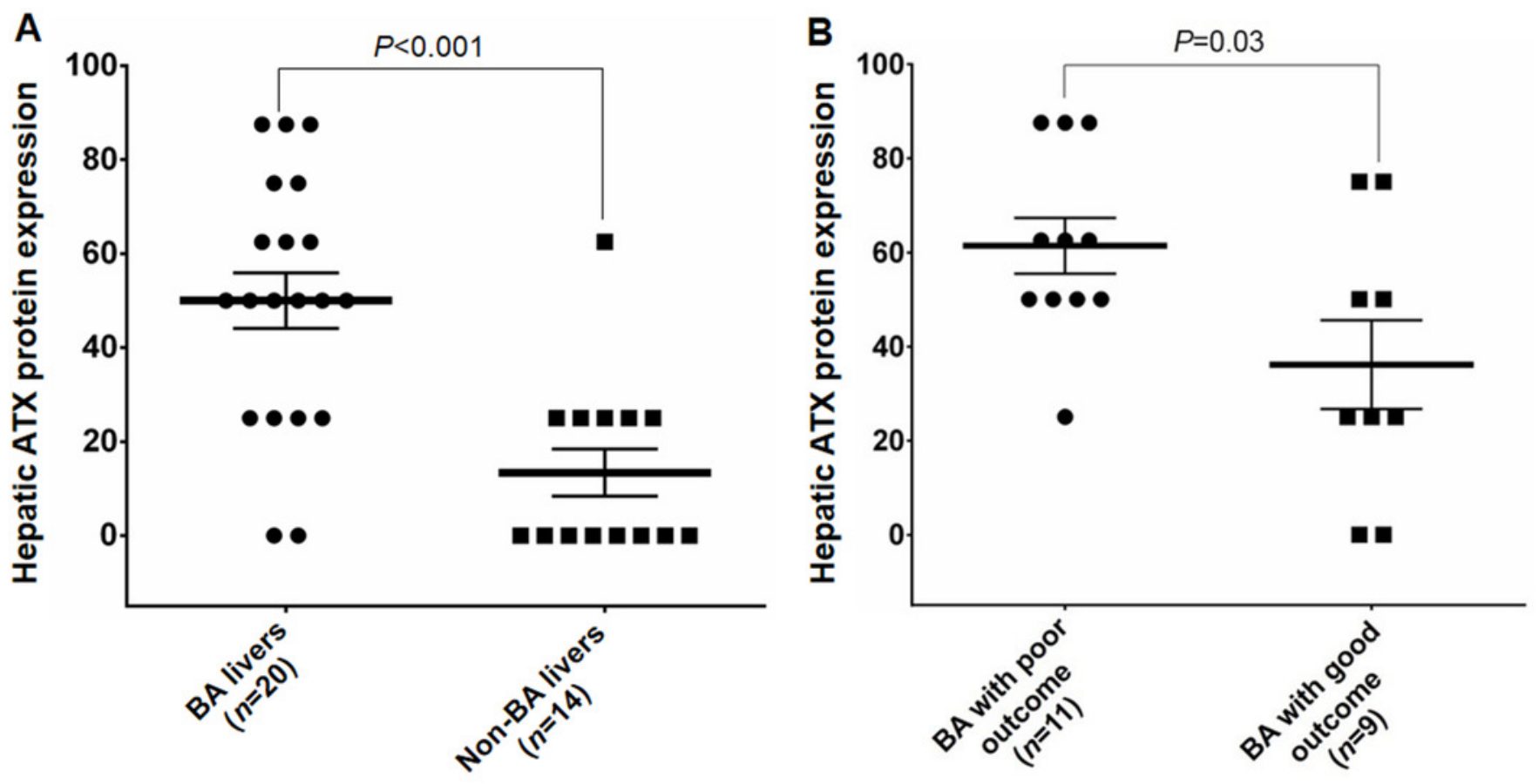


\section{Figure 4}

Hepatic ATX expression and liver fibrosis.

(A) Histopathological analysis of liver samples in BA (Masson Trichrome staining,

magnifications 100x). (B) Hepatic ATX protein expression correlated positively with Metavir fibrosis stage in $\mathrm{BA}(r=0.79, P<0.001)$.
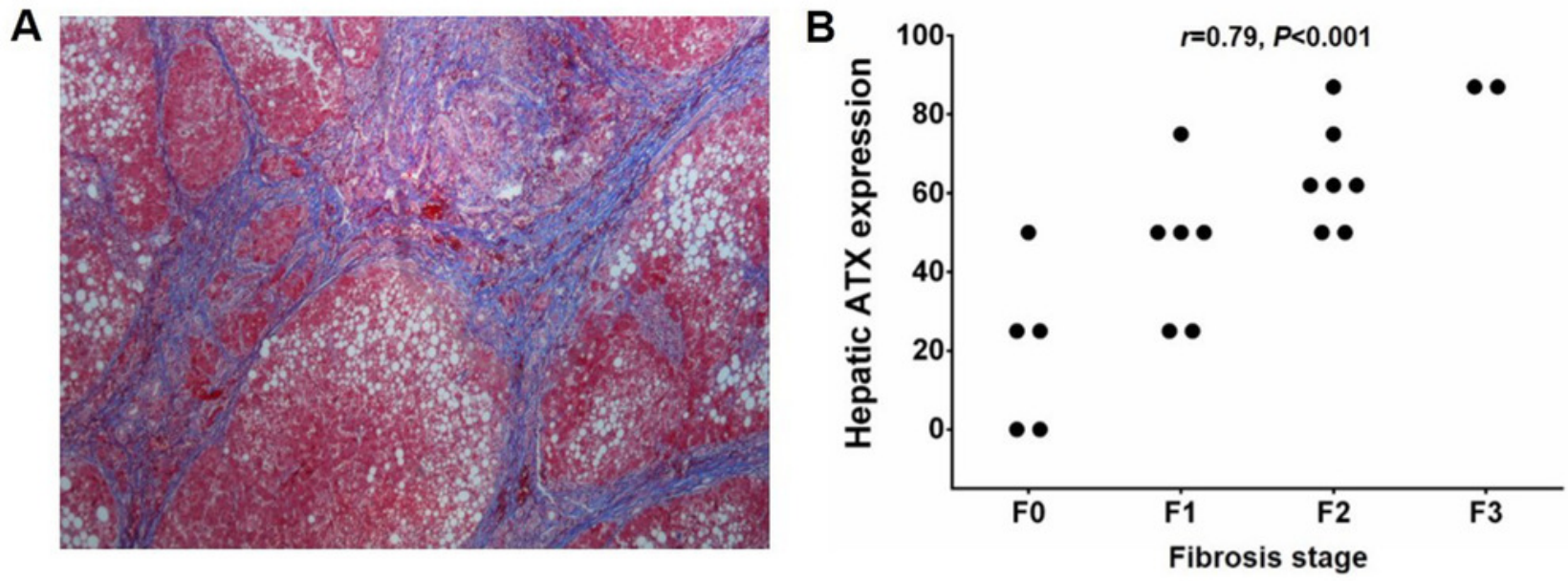


\section{Figure 5}

Kaplan-Meier survival curve of BA children after KPE over 10 years.

(A) The overall survival curve of 20 BA reveals that 10 -year survival rates with native livers are $80 \%$. (B) Survival curve comparisons display that BA children with low ATX expression $(n=12)$ have 10-year survival greater than those with high ATX expression $(n=8)$ (log-rank, $\left.X^{2}=2.17, P=0.14\right)$.
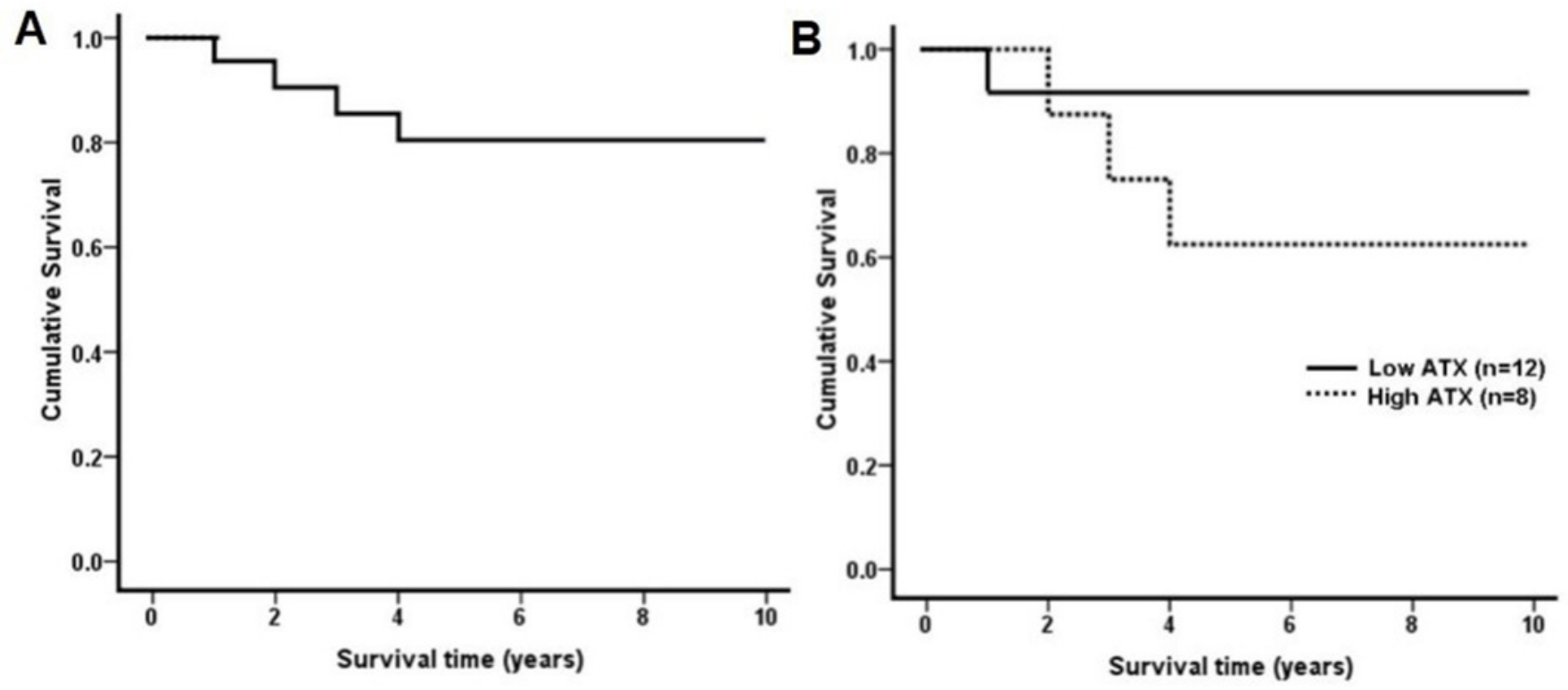


\section{Table $\mathbf{1}$ (on next page)}

Demographic and clinical characteristics of BA patients and non-BA controls.

Data presented as mean \pm SEM. $P$-value $<0.05$ indicates a statistically significant difference in clinical data between BA patients and non-BA controls at the time of Kasai portoenterostomy (KPE). Abbreviations: BA, biliary atresia; ALT, alanine aminotransferase; NA, data not available; SEM, standard error of the mean. 
1

\begin{tabular}{|l|l|l|l|}
\hline Characteristics & $\begin{array}{l}\text { BA patients } \\
(n=20)\end{array}$ & Non-BA controls & P-value \\
\hline Age (days) & $91.1 \pm 7.0$ & $897.5 \pm 315.2$ & \\
\hline Gender (female:male) & $12: 8$ & $8: 6$ & 0.6 \\
\hline Albumin (g/dL) & $4.1 \pm 0.1$ & $4.2 \pm 0.3$ & 0.3 \\
\hline Total bilirubin (mg/dL) & $12.2 \pm 0.8$ & NA & - \\
\hline ALT (IU/L) & $191.8 \pm 25.8$ & NA & - \\
\hline Hepatic ATX expression (\%) & $50.0 \pm 5.9$ & $13.4 \pm 5.0$ & $<0.001$ \\
\hline
\end{tabular}

2

3 Note.

4 Data presented as mean \pm SEM.

$5 \quad P$-value $<0.05$ indicates a statistically significant difference in clinical data between BA patients

6 and non-BA controls at the time of Kasai portoenterostomy (KPE).

7 Abbreviations: BA, biliary atresia; ALT, alanine aminotransferase; ATX, autotaxin; SEM,

8 standard error of the mean.

9

10

11 


\section{Table 2 (on next page)}

Demographic and clinical characteristics of BA patients based on clinical outcome at 6 months post-Kasai.

Data presented as mean \pm SEM. $P$-value $<0.05$ indicates a statistically significant difference in clinical data between BA patients with poor outcome and good outcome at 6 months postKasai. Abbreviations: BA, biliary atresia; ALT, alanine aminotransferase; NA, data not available; SEM, standard error of the mean. 
1

\begin{tabular}{|l|l|l|l|}
\hline Characteristics & BA patients with poor & BA patients with good & $P$-value \\
outcome $(n=11)$ & outcome $(n=9)$ & \\
\hline Age at operation (days) & $98.2 \pm 11.3$ & $77.0 \pm 4.9$ & 0.1 \\
\hline Gender (female:male) & $6: 5$ & $6: 3$ & 0.4 \\
\hline Albumin (g/dL) & $3.5 \pm 0.2$ & $4.1 \pm 0.1$ & $<0.05$ \\
\hline Total bilirubin (mg/dL) & $5.7 \pm 1.7$ & $0.4 \pm 0.1$ & $<0.001$ \\
\hline ALT (IU/L) & $140.2 \pm 27.2$ & $82.8 \pm 16.2$ & 0.01 \\
\hline Hepatic ATX expression (\%) & $61.4 \pm 5.9$ & $36.1 \pm 9.4$ & 0.03 \\
\hline
\end{tabular}

2

3 Note.

4 Data presented as mean $\pm \mathrm{SEM}$.

$5 \quad P$-value $<0.05$ indicates a statistically significant difference in clinical data between BA patients

6 with poor outcome and good outcome at 6 months post-Kasai.

7 Abbreviations: BA, biliary atresia; ALT, alanine aminotransferase; ATX, autotaxin; SEM,

8 standard error of the mean.

9 